\title{
SISTEMA COM ATMOSFERA CONTROLADA PARA A CARACTERIZAÇÃo DE SENSORES A GASES
}

Ricardo L. T. Andrade, Cleber A. Lindino e Luis Otavio de Sousa Bulhões

Departamento de Química - Universidade Federal de São Carlos - CP 676 - 13565-905 - São Carlos - SP

Recebido em 29/4/97; aceito em 14/8/97

\begin{abstract}
CONTROLLED ATMOSPHERE SYSTEM FOR GAS SENSORS CHARACTERIZATION. This paper describes a sample holder for the electrical measurement of oxides or conducting polymers in the form of pellets or films which are used as gas sensors. The system makes it possible to control the sample temperature, the gas pressure and composition. The temperature in the sample can be changed from $25^{\circ} \mathrm{C}$ to $450^{\circ} \mathrm{C}$, and the gas pressure in the chamber is controlled between $5 \times 10^{-4}$ and 1000 mbar. The performance of the system in resistance measurements of doped tin oxide pellets and polyaniline films deposited on platinum electrodes for methane is analyzed.
\end{abstract}

Keywords: gas chamber; gas sensors; oxides; conducting polymers.

\section{INTRODUÇÃO}

Durante a última década, inúmeros transdutores foram desenvolvidos para a utilização como sensores químicos, especialmente sensores a gases, a vapores orgânicos e odores ${ }^{1-5}$. O estudo das propriedades físicas destes materiais, para uso como sensores, necessita de condições controladas para avaliar parâmetros como sensibilidade, seletividade, reprodutibilidade e tempo de vida. A reprodutibilidade e a precisão das medidas exigem sistemas com controle preciso da atmosfera de trabalho e da temperatura.

Os sistemas para a caracterização física de sensores descritos na literatura ${ }^{6-7}$ são pouco adequados para estudos analíticos pois apresentam deficiências que prejudicam a confiabilidade dos resultados. As principais deficiências são: falta de controle da pressão total do gás (ou gases) no interior da câmara, prejudicando a determinação da fugacidade do gás; ausência de um sistema para mistura de gases, pois esta condição é importante na determinação de interferentes; câmaras com volumes superiores a $20 \mathrm{~L}$ o que dificulta a desorção dos gases da superfície do sensor, processo fundamental para a reprodutibilidade do sensor. Além disso, estes sistemas possuem grandes dimensões que dificultam o manuseio.

Neste trabalho descreve-se a construção de uma câmara para a caracterização física de sensores com atmosfera controlada, versátil, simples, com baixo nível de consumo de gases e de fácil manutenção. Este sistema tem sido utilizado em nosso laboratório para testes de sensores a gases construídos a partir de óxidos semicondutores ou de polímeros condutores.

\section{DESCRIÇÃO DO SISTEMA}

A figura 1 mostra uma fotografia do conjunto utilizado como sistema para as medidas de resistência de sensores a temperatura e atmosfera controlada. O sistema consiste de uma câmara cilíndrica (C) fabricada em latão, com $225 \mathrm{~mm}$ de comprimento por $94 \mathrm{~mm}$ de diâmetro. Em uma das extremidades, a câmara possui uma flange de $150 \mathrm{~mm}$ de diâmetro, utilizada como tampa e suporte do porta-amostra, que é apresentado em detalhes na figura 2. Esta flange e um O-ring são fixos à câmara com o auxílio de seis parafusos. Na parte externa da tampa foram fixados seis conectores do tipo BNC. Dois conectores são utilizados para a alimentação da resistência usada no aquecimento do sensor (Figura 2). Dois conectores são usados para a ligação entre o termopar ( $\mathrm{Cu} /$ Constantan) e o controlador de temperatura (Gefran modelo 500); finalmente, dois conectores são utilizados para as medidas de resistência do sensor. A tampa possui ainda, em sua parte interna, um suporte para acomodar o sensor sobre a resistência durante as medidas.

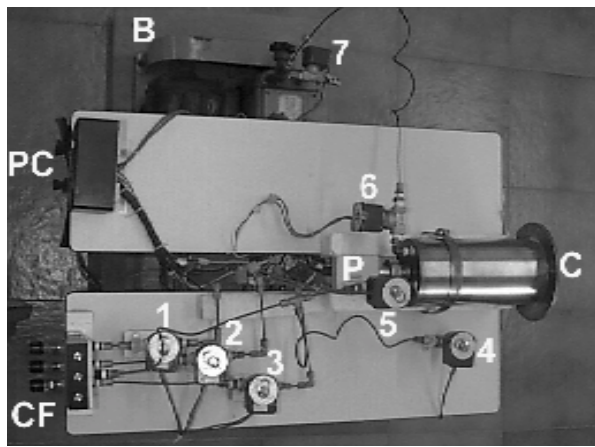

Figura 1. Fotografia do sistema para a caracterização física de sensores a gás; $(C)$ câmara; $(P)$ sensor de pressão; $(B)$ bomba de vácuo; (CF) controlador de fluxo; (PC) painel de controle; (1-7) válvulas solenóides.

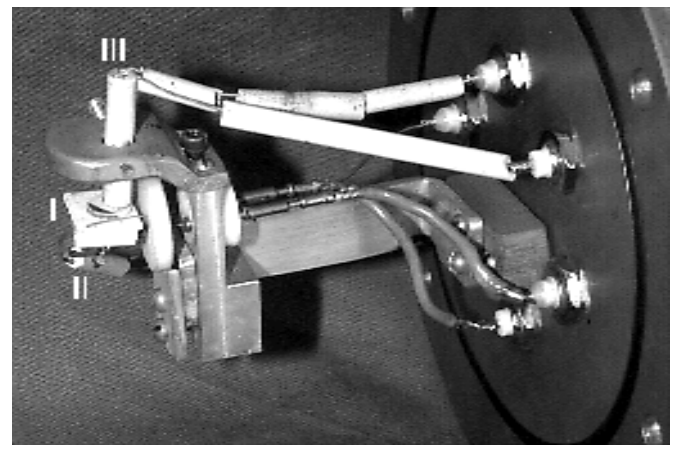

Figura 2. Fotografia do porta-amostra.

A outra extremidade da câmara possui também uma flange, de $30 \mathrm{~mm}$ de diâmetro onde acoplou-se um sensor de pressão tipo Pirani Balzers modelo TPR 250 (P) adequado para realizar medidas de pressão na faixa entre $5 \times 10^{-4}$ e 1000 mbar, e dois orifícios aos quais foram conectadas válvulas solenóides (Ascoval) de duas vias (1 a 7), normalmente fechadas (as válvulas se encontram fechadas quando desenergizadas), que 
controlam a entrada e saída de gases do sistema. O controle das válvulas é feito em um painel (PC). A válvula de saída (6) está conectada a uma bomba de vácuo (B), utilizada para facilitar a exaustão de gases no interior da câmara e, conseqüentemente, promover a limpeza do material. A válvula (7) serve para quebrar o vácuo. A válvula de entrada (5) está conectada a uma linha de gás, construída em tubos de cobre de $1 / 8$ " de diâmetro, onde as válvulas (1), (2) e (3) controlam a passagem de gás dos cilindros para os controladores de fluxo.

O sistema de controle de fluxo (CF), da Cole Parmer, consiste de um suporte de alumínio com três entradas em paralelo, onde cada gás passa por um tubo de vidro conectado a uma válvula manual de alta resolução que permite o controle do fluxo. O controlador de fluxo possui uma saída em comum, que possibilita a mistura de gases. O fluxo pode ser controlado nas faixas entre 28 a $1249 \mathrm{~mL} / \mathrm{min}, 13,1$ a $202,1 \mathrm{~mL} / \mathrm{min}$ e 2,8 a $48,7 \mathrm{~mL} / \mathrm{min}$. Estes medidores podem ser substituídos propiciando diferentes fluxos. Entre o controlador de fluxo e a câmara existe um divisor que permite ajustar a composição da mistura dos gases, antes de injetá-la na câmara.

$\mathrm{O}$ funcionamento do sistema pode ser resumido da seguinte forma: os gases provenientes dos cilindros passam pelas válvulas (1), (2) ou (3), entram em paralelo pelo sistema de controle de fluxo, saindo por um tubo comum que permite a mistura dos gases, sendo o controle da mistura realizado com o auxílio da válvula (4). Controlada a proporção dos gases, a válvula (4) é fechada e a mistura é injetada na câmara. As medidas podem ser estáticas ou em fluxo, bastando abrir, ou não, a válvula (6). A pressão interna da câmara é controlada pelo sensor Pirani e a temperatura do sensor é medida através de um termopar.

O porta-amostra pode ser observado na figura 2 , onde há um sistema de aquecimento (I), operando na faixa entre 25 a $450^{\circ} \mathrm{C} \pm 0,5$, o termopar para o controle da temperatura do sensor (II) e as pontas de contato (III) para a realização de medidas elétricas de duas pontas, utilizando contatos de platina.

As medidas de resistência elétrica foram realizadas com um multímetro Hewlett Packard modelo 34401 A.

\section{TESTE DE FUNCIONAMENTO}

A câmara foi utilizada na caracterização de sensores a gases baseados em óxidos semicondutores e polímeros condutores. A figura 3 mostra a repetibilidade de medidas de resistência elétrica de um sensor ao gás metano, baseado em um polímero condutor, a polianilina, com erro relativo de $2 \%$ e desvio padrão de 0,04 . Este erro relativo de $2 \%$ corresponde ao erro relativo do fundo de escala do fluxímetro utilizado. O sensor construído com o filme de polianilina apresenta resposta linear em fugacidade entre $1,2 \mathrm{e}$ $2,4 \times 10^{-3}$ atm $(0,25 \%$ e $0,6 \% \mathrm{v} / \mathrm{v})$ de concentração de gás metano, com coeficiente de correlação de 0,998 e desvio padrão de 0,012 (média de cinco medidas), como pode ser observado na figura 4 .

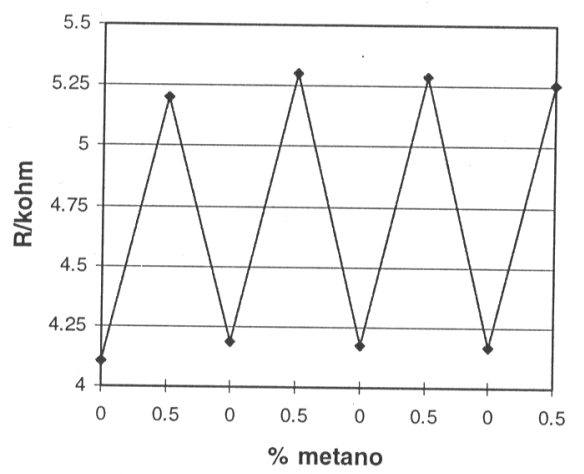

Figura 3. Resposta do sensor baseado em polianilina a pulsos de gás metano $(0,5 \%$ em nitrogênio $v / v), T=25^{\circ} \mathrm{C}$, utilizando-se o sistema de medidas descrito.

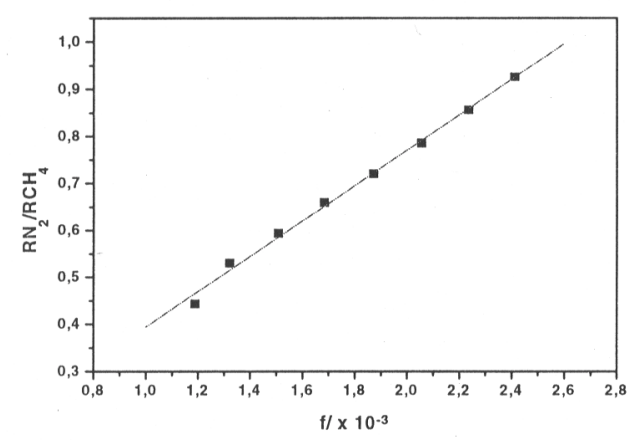

Figura 4. Resposta linear do sensor baseado em polianilina ao gás metano, onde $\mathrm{RN}_{2} / \mathrm{RCH}_{4}$ é a normalização das medidas em relação ao gás de referência (nitrogênio), $T=25^{\circ} \mathrm{C}$.

A figura 5 mostra a sensibilidade ao gás metano de um sensor de óxido de estanho dopado com 5,0\% de antimônio em função da temperatura de trabalho. O sistema permite o controle da temperatura de trabalho do sensor com precisão de $0,5^{\circ} \mathrm{C}$ apresentando boa estabilidade no decorrer das determinações, assegurando que as variações de resistência do sensor observadas são decorrentes de trocas eletrônicas entre o gás e o semicondutor, e não de variações na temperatura. A resposta deste sensor a diferentes quantidades de $\mathrm{CH}_{4}$ pode ser observada através da figura 6 . O sensor apresentou uma resposta linear na faixa de 0,15 a $0,35 \%$ de $\mathrm{CH}_{4}$ misturado com ar sintético, com coeficiente de correlação de 0,99 e desvin nadrão de $375 \%$

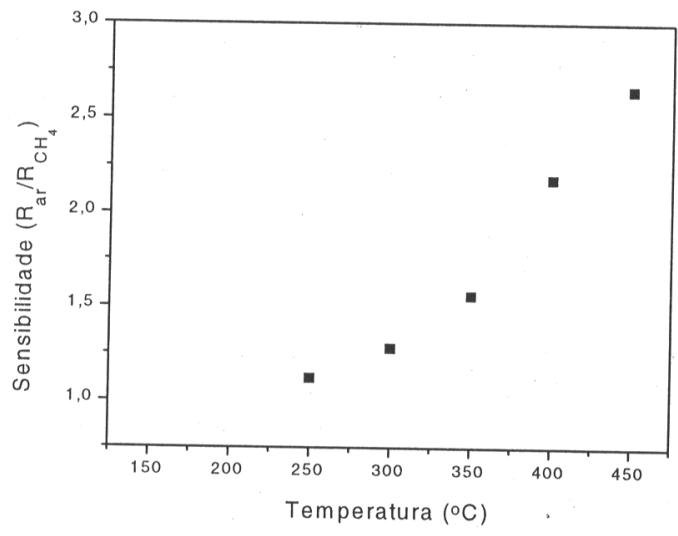

Figura 5. Variação da sensibilidade em função da temperatura de trabalho para o $\mathrm{SnO}_{2}$ dopado com 5,0\% de Sb, submetido a uma atmosfera com 2,0\% v/v de metano

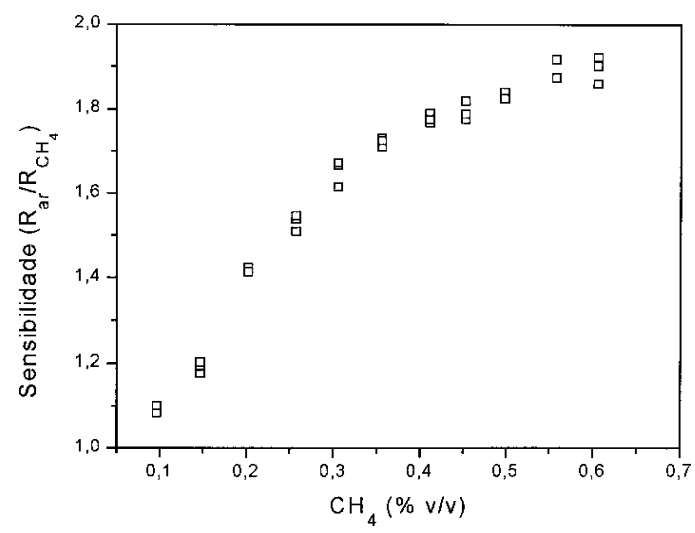

Figura 6. Sensibilidade como função da quantidade de metano na atmosfera para o $\mathrm{SnO}_{2}$ dopado com 5,0\% de $\mathrm{Sb}$. Temperatura $450^{\circ} \mathrm{C}$. 


\section{CONCLUSÕES}

O sistema construído permite o controle da temperatura do sensor, da pressão no interior da câmara, além de possibilitar a realização de misturas gasosas compostas por até três diferentes gases, com composição controlada. Devido a sua versatilidade, o sistema pode ser utilizado no desenvolvimento de sensores a gases e em estudos do comportamento elétrico de materiais em diferentes atmosferas.

\section{AGRADECIMENTOS}

Os autores agradecem aos técnicos José Roberto Vosenaar e Florentino Flori Jr. pela ajuda na construção da câmara de testes e ao CNPq, CAPES e FAPESP pelo apoio financeiro.

\section{REFERÊNCIAS}

1. Gopel, W.; Sensors and Actuators B 1994, 18-19, 1.

2. Gardner, J. W., Bartlett, P. N.; Sensors and Actuators B 1994, 18-19, 211.

3. MartinellI, G.;Carotta, M. C.; Sensors and Actuators, B23: 1995, 157.

4. Löw, H.; Sulz, G.; Lacher, M.; Külner, G.; Uptmoor, G.; Reiter, H.; Steiner, K.; Sensors and Actuators, B9: 1992, 215.

5. Feng, C. D.; Shimizu, Y.; Egashira, M.; J. Eletrochem. Soc. 1994, 141, 220.

6. Mousa-Bahia, A. A., Coles, G. S. V., Watson, J.; Sensors and Actuators B 1993, 12, 141.

7. Nelson, G.; Gas Mixtures: Preparation and Control; Lewis Publishers, California, 1992. 